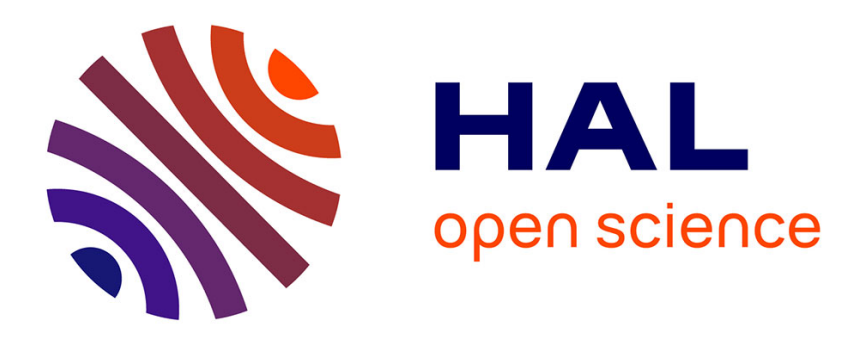

\title{
Suicide des personnes écrouées en France: évolution et facteurs de risque
}

Géraldine Duthé, Angélique Hazard, Annie Kensey

\section{To cite this version:}

Géraldine Duthé, Angélique Hazard, Annie Kensey. Suicide des personnes écrouées en France: évolution et facteurs de risque. Population (édition française), 2015, 69 (4), pp.519-549. 10.3917/popu.1404.0519 . hal-03130788

\section{HAL Id: hal-03130788 \\ https://hal.science/hal-03130788}

Submitted on 3 Feb 2021

HAL is a multi-disciplinary open access archive for the deposit and dissemination of scientific research documents, whether they are published or not. The documents may come from teaching and research institutions in France or abroad, or from public or private research centers.
L'archive ouverte pluridisciplinaire HAL, est destinée au dépôt et à la diffusion de documents scientifiques de niveau recherche, publiés ou non, émanant des établissements d'enseignement et de recherche français ou étrangers, des laboratoires publics ou privés. 


\title{
Géraldine DuthÉ*, Angélique HAZARD**, Annie KENSEY**
}

\section{Suicide des personnes écrouées en France : évolution et facteurs de risque}

\begin{abstract}
L'univers carcéral est-il propice au suicide? L'est-il davantage aujourd'hui? Dans un article de Population paru il y a près de 40 ans, Jean-Claude Chesnais établissait pour la France une nette sursuicidité des personnes détenues par rapport à la population libre. À partir des données administratives de la direction de l'administration pénitentiaire du ministère de la Justice, Géraldine DUTHÉ, Angélique HAZARD et Annie KENSEY mettent à leur tour en évidence la sursuicidité de la population masculine écrouée par rapport à la population générale. Tandis que les taux de suicide ont relativement peu varié au cours du temps dans la population générale, ils n'ont cessé d'augmenter en prison et y sont aujourd'hui sept fois plus fréquents qu'en milieu libre. Analysant le suicide des personnes écrouées entre 2006 et 2009, les auteurs identifient les principaux facteurs de risque liés à la condition carcérale.
\end{abstract}

Près de la moitié des décès survenus dans les prisons françaises sont des suicides, et le niveau de mortalité associé y est bien plus élevé qu'en population générale (Duthé et al., 2009; Aebi et Delgrande, 2010). La spécificité des prisons et la vulnérabilité des détenus face au suicide sont depuis longtemps mises en avant en France (Chesnais, 1976; Tournier et Chemithe, 1979; Kensey, 1991; Bourgoin, 1993a) et dans d'autres pays (Kariminia et al., 2007; Fazel et al., 2010). Le taux de suicide en prison a globalement beaucoup augmenté durant les dernières décennies. Il était en France au milieu des années 2000 le plus élevé des pays d'Europe de l'Ouest ${ }^{(1)}$ (Aubusson de Cavarlay, 2009; Duthé et al., 2009).

Les facteurs liés au suicide en prison sont distincts de ceux de la population générale, en raison des conditions très particulières dans lesquelles ils surviennent. Plusieurs études ont montré que l'incarcération était un moment

(1) Sans tenir compte du Luxembourg où le taux, calculé sur de très faibles effectifs (entre 0 et 3 décès annuels au milieu des années 2000), fluctue fortement d'une année à l'autre.

* Institut national d'études démographiques.

** Direction de l'administration pénitentiaire.

Correspondance : Géraldine Duthé, Institut national d'études démographiques, 133 boulevard Davout, 75980 Paris Cedex 20, tél : 33 (0)1 560622 47, courriel : geraldine.duthe@ined.fr 
à risque élevé et que les prévenus se suicidaient plus que les condamnés ${ }^{(2)}$. Parmi les suicidés, les personnes condamnées pour des infractions violentes et sexuelles sont surreprésentées tout comme celles condamnées à de longues peines. Du point de vue des conditions d'incarcération, la fréquence de suicides est plus élevée chez les détenus qui sont seuls dans leur cellule ou placés en cellule disciplinaire. Parmi les facteurs sociodémographiques, il n'existe pas de consensus sur l'effet de l'âge. Par ailleurs, même si la sursuicidité des femmes en prison par rapport au milieu libre est bien supérieure à celle des hommes (Bourgoin, 1993a), ces derniers se suicident plus que les femmes. En prison, les célibataires se suicident moins que les personnes mariées alors qu'on observe l'inverse en milieu libre (Durkheim, 1897). Les personnes en couple doivent en effet gérer une rupture supplémentaire avec la séparation et l'éloignement de leur conjoint-e $e^{(3)}$. Des différences selon l'origine ethnique sont constatées, dans les pays où des données existent, avec un risque plus élevé dans le groupe majoritaire. Enfin, les troubles mentaux et du comportement, plus fréquents chez les détenus que dans la population générale (Fazel et Danesh, 2002; Mouquet, 2005; Falissard et al., 2006), sont aussi fortement associés au risque de suicide ${ }^{(4)}$.

Les études statistiques sur ce sujet sont difficiles à mener en raison de l'accès limité aux informations individuelles des personnes sous écrou d'une part, et du fait que le suicide est un phénomène statistiquement peu fréquent d'autre part. De ce fait, la plupart des études se basent soit sur une comparaison agrégée des caractéristiques des personnes qui se sont suicidées par rapport à la population en prison, soit sur des périodes d'observation longues, soit encore sur des comparaisons avec un groupe « témoin ». Rares sont les études statistiques multivariées conduites au niveau individuel et exhaustives, permettant d'estimer les effets propres de chacun des différents facteurs de risque. Pour contribuer à mieux appréhender le phénomène du suicide en prison en France, des informations ont été collectées à partir de la base nationale de gestion informatisée des détenus (Gide) de l'administration pénitentiaire. Elles portent

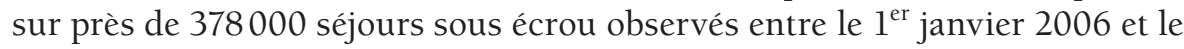
15 juillet 2009 en France et permettent d'étudier les facteurs de risque du suicide au cours de cette période durant laquelle 378 suicides ont été identifiés. La disponibilité des dates des différents événements nous permet d'explorer les risques différentiels en considérant la durée d'exposition au risque de se

(2) La plupart des études sont multifactorielles. On trouvera une synthèse des facteurs du suicide en prison dans Fazel et al. (2008). Par ordre chronologique, les études auxquelles nous faisons référence ici sont : Dooley, 1990; Marcus et Alcabes, 1993; DuRand et al., 1995; Joukamaa, 1997; Frottier et al., 2002; Fruehwald et al., 2004; Shaw et al., 2004; Way et al., 2005; O'Driscoll et al., 2007. Pour le contexte français : Chesnais, 1976; Bourgoin, 1993a, 1993b; Guillonneau, 2002; Hazard, 2008. Pour le détail par facteur, voir Duthé et al., 2013.

(3) Plus d'un détenu sur dix vit une séparation dans le premier mois de détention (Cassan et al., 2000).

(4) Le lien entre santé mentale et suicide en prison a été mis en avant dans de nombreux travaux : Marcus et Alcabes, 1993; Joukamaa, 1997; Towl et Crighton, 1998; Fruehwald et al., 2003; Shaw et al., 2004; Way et al., 2005 ; Fazel et al., 2008; Hawton et van Heeringen, 2009; Fazel et Baillargeon, 2011. 
suicider. Elle permet aussi de tenir compte de possibles changements survenus pour la personne écrouée, comme son changement de situation pénale ou de ses conditions d'incarcération.

Comment a évolué le suicide en prison en France? Quels facteurs contribuent au suicide? Dans cet article, nous proposons en premier lieu de revenir sur l'évolution du suicide en prison observable depuis le milieu du XIX ${ }^{e}$ siècle, en soulignant la distinction avec le suicide en population générale. La seconde partie explore les associations qui existent entre les risques de se suicider et les caractéristiques individuelles de personnes écrouées dans une période récente. L'identification de ces associations nous permet de discuter des facteurs en cause, dans le contexte des politiques actuellement mises en place pour prévenir les suicides dans les prisons françaises.

\section{Le suicide en prison : perspective historique}

\section{Données et population}

Les données utilisées dans cette partie proviennent de deux sources : celles antérieures à 1945 sont les séries publiées par période (de deux à cinq années) par Jean-Claude Chesnais en 1976. Les données ultérieures, annuelles, sont celles publiées ou mises à disposition par la direction de l'administration pénitentiaire (DAP). L'ensemble de ces séries font référence à la population sous écrou en France métropolitaine. Jusqu'au milieu des années 2000, très peu de personnes étaient écrouées sans être détenues en milieu carcéral, ces deux populations (écrouée et carcérale) sont donc tout à fait similaires. Le placement sous surveillance électronique s'est développé à partir de 2004 et la proportion des individus écroués non détenus est passée de moins de $2 \%$

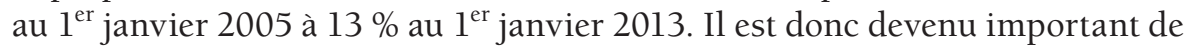
faire la distinction entre ces deux populations (écrouée et carcérale).

\section{Évolution de la population écrouée depuis le milieu du XIXe siècle}

En 1852-1855, les prisons françaises comptaient 25467 personnes en moyenne, mais la population augmente de la fin des années 1860 jusqu'au milieu des années 1870 et on dénombre près de 44000 détenus durant la période 1872-1875. Malgré une hausse autour de la première guerre mondiale, les effectifs baissent globalement jusqu'à la veille de la seconde guerre mondiale $^{(5)}$. En 1936-1938, on compte ainsi un peu plus de 15000 détenus (Chesnais, 1976). Les statistiques ne sont pas disponibles pour la période de la guerre, mais 1945 est une année record avec plus de 63000 détenus, soit quatre fois plus qu'avant la guerre. À cette époque, beaucoup sont en prison pour faits de collaboration et sont libérés dans les années suivantes. Les effectifs diminuent

(5) À l'époque, de nombreux détenus partaient en relégation en Guyane et en Nouvelle-Calédonie. Envoyés au bagne, ils sortaient de la statistique de la population écrouée en France métropolitaine. 
rapidement jusqu'en 1955 où moins de 20000 personnes sont sous écrou en moyenne. Depuis, malgré les fluctuations annuelles, l'effectif a sensiblement augmenté et le nombre de personnes écrouées en 2010 atteint près de 62000 (figure 1; tableau annexe A.1).

Figure 1. Effectifs annuels de la population moyenne sous écrou depuis le milieu du XIX ${ }^{e}$ siècle en France métropolitaine

Par période jusqu'en 1938

(Chesnais, 1976)

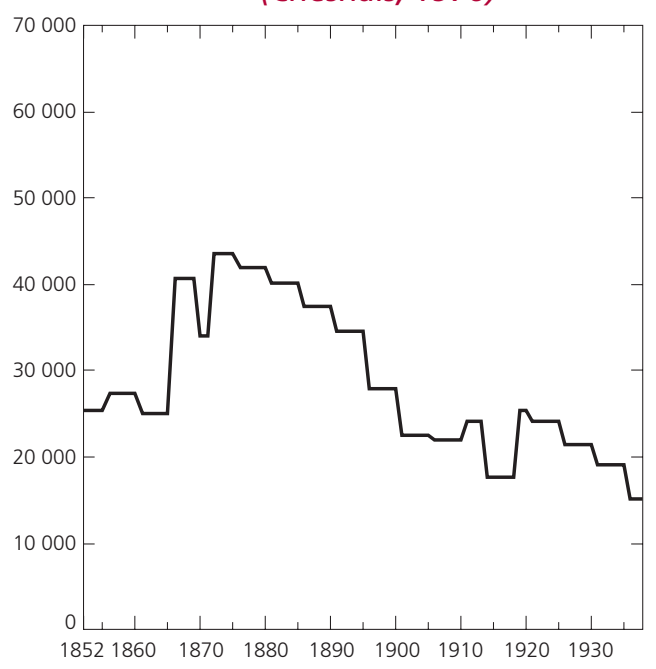

Par année à partir de 1944 (données DAP)

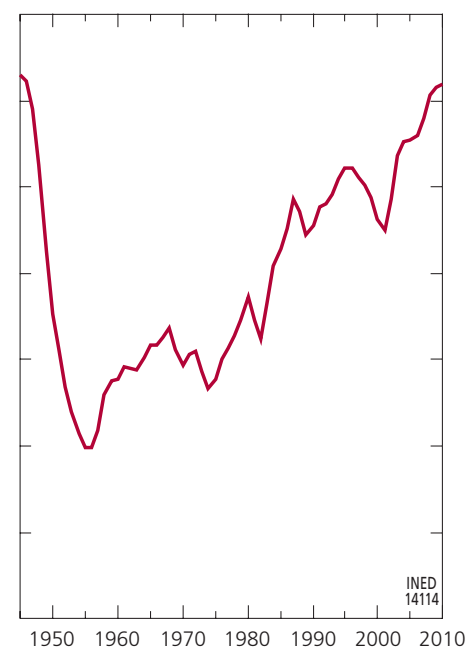

Sources : Chesnais (1976); Ministère de la Justice, DAP.

La seconde moitié du $\mathrm{XX}^{\mathrm{e}}$ siècle est donc marquée par une forte croissance qui aboutit à une surpopulation carcérale. En 1990, le taux d'occupation ${ }^{(6)}$ atteint ainsi 124 détenus pour 100 places. Au début des années 2010, la situation s'améliore un peu avec les différents programmes de construction d'établissements pénitentiaires, mais on dénombre toujours 113 détenus pour 100 places (DAP, 2012).

Au niveau individuel, le profil des détenus a beaucoup changé. Les évolutions législatives modifient la composition de la population pénitentiaire (Fruehwald et al., 2002). Les changements de la législation pénale sont le produit d'une moindre tolérance de la société à certaines infractions : les dispositions pénales se sont multipliées et les peines prononcées ont tendance à s'allonger. Certains dispositifs modifient le profil pénal des personnes écrouées : la loi de 1980 donnant pour la première fois une définition des éléments constitutifs du crime de viol amène à l'incarcération d'auteurs de

(6) Le taux d'occupation, ou densité carcérale, mesure à une date donnée, la proportion de personnes détenues par rapport au nombre de places opérationnelles des établissements. Lorsque cet indicateur dépasse $100 \%$, il y a moins en places de détention que de détenus. On parle alors de surpopulation carcérale. 
différents actes dorénavant juridiquement définis comme des viols; la mise en place d'un débat contradictoire obligatoire avant toute mise en détention provisoire en 1984 a pour effet de diminuer la part des prévenus; l'allongement en 1989 de la durée de prescription des victimes mineures d'agression sexuelle fait augmenter le nombre de personnes écrouées pour ces infractions; dans les années 2000, l'élargissement de la définition pénale d'une violence volontaire fait augmenter le nombre de personnes écrouées pour ce type d'infraction; etc.

En parallèle, le profil démographique des détenus a quelque peu évolué. En 1852, année de début de période, on dénombrait $17 \%$ de femmes parmi les prévenus $^{(7)}$. À la fin de la seconde guerre mondiale, la proportion de femmes dans la population écrouée est à peu près la même (18\% en 1946) mais la part des femmes baisse rapidement par la suite. Depuis 1958, elle reste inférieure à 5 \% (Duthé et al., 2011). Par ailleurs, la population sous écrou a vieilli, l'âge moyen est actuellement autour de 34 ans, mais la standardisation -qui a pu être menée sur les trois dernières décennies - ne modifie pas les tendances du suicide en prison (Duthé et al., 2010).

Concernant l'état de santé de la population carcérale, les soins des personnes détenues sont pris en charge par le ministère de la Santé et les hôpitaux publics depuis 1994 et le dispositif sanitaire s'est depuis nettement amélioré. Cependant, les besoins restent très élevés et insuffisamment satisfaits (Coldefy et al., 2002; Falissard et al., 2006; Coldefy et Exertier, 2007), une situation que l'on observe d'ailleurs au niveau international (Ashraf, 1999; Fazel et Baillargeon, 2011).

Concernant plus spécifiquement la santé mentale des détenus, il est difficile d'en connaître l'évolution au fil du temps (tout comme en population générale). Il faut remonter à l'année 1838 pour voir apparaître la première loi de prise en charge des malades mentaux -auparavant placés en prison quand leur comportement troublait l'ordre social-, avec l'ouverture d'établissements d'aliénés sur le territoire français, qui seront ensuite remplacés par des hôpitaux psychiatriques (Mamelet, 1978, cité par Coldefy, 2007). Cependant, ces hôpitaux ont isolé les malades de la société, les rendant vulnérables, exposés à la négligence voire aux mauvais traitements. Associées au manque d'hygiène et de soins, les restrictions alimentaires durant la seconde guerre mondiale ont conduit à une surmortalité dramatique dans les hôpitaux psychiatriques avec plus de 45000 personnes qui y sont décédées pendant la guerre (Chapireau, 2007; von Bueltzingsloewen, 2007). Face à cette hécatombe, mais aussi avec la prise de conscience de l'horreur concentrationnaire après la guerre et l'arrivée des neuroleptiques permettant de réguler certains troubles dans les années 1950, une désinstitutionalisation du secteur psychiatrique s'est amorcée. Ce mouvement s'est caractérisé par le développement de l'hospitalisation libre et de la délivrance des soins en ambulatoire, qui deviendront sectorisés dans les années 1970 (Coldefy, 2007). Cette ouverture du secteur psychiatrique vers plus

(7) D’après le compte général de l'administration de la justice criminelle en France pour l'année 1852 disponible sur le site Gallica de la BNF (http://gallica.bnf.fr/). 
d'humanisation s'est aussi traduite par des changements dans les priorités et les moyens associés. Ainsi, le nombre de lits hospitaliers a diminué. Ce serait l'une des raisons invoquée pour affirmer une présence accrue de malades mentaux en prison. La seconde, également liée à cette évolution, est l'évaluation de la responsabilité pénale : dans le cas d'une personne souffrant de troubles psychologiques, cette responsabilité peut être effective malgré une « altération du discernement $»$. Toutefois, le lien encore souvent affirmé entre prison et psychiatrie qui pourrait expliquer en partie la hausse des suicides observée en prison n'est pas statistiquement établi, et aucune étude ne permet de l'attester.

\section{Hausse du taux de suicide en prison depuis le milieu du XIX siècle}

Le nombre annuel de suicides en prison regroupe l'ensemble des décès consécutifs à un acte suicidaire des personnes écrouées au moment de cet acte. Ce nombre de suicides est rapporté à la population moyenne écrouée de l'année (ou de la période considérée) pour obtenir un taux brut de suicide qui est disponible depuis le milieu du XIX ${ }^{\mathrm{e}}$ siècle pour le territoire hexagonal. Le taux est calculé pour plusieurs années jusqu'en 1938, ce qui masque les variations annuelles, il n'est pas disponible pour la période 1939-1944, et nous ne disposons de statistiques annuelles que depuis 1945 (à partir desquelles nous pouvons aussi calculer un taux par période quinquennale $)^{(8)}$.

Le taux de suicide en prison a beaucoup fluctué depuis le milieu du XIX ${ }^{e}$ siècle : le taux le plus faible est observé en 1946 (1,4 suicide pour 10000 personnes écrouées), et le plus élevé en 1996 (26 suicides pour 10000). Outre les fluctuations annuelles, on observe des périodes de creux (durant la décennie 1855-1865 et au lendemain de la seconde guerre mondiale) et de pics (durant la décennie 1905-1915, dans le milieu des années 1950, de la fin des années 1990 au début des années 2000). Au-delà de ces fluctuations, une tendance nette de la progression du taux de suicide en prison se dégage au cours du temps : il est ainsi passé de 5 pour 10000 en $1852-1855$ à 18,5 pour 10000 pour la période 2005-2010 (figure 2).

Actuellement, plus de neuf détenus sur dix sont des hommes âgés de 15 à 60 ans, le taux brut de suicide en prison peut donc être comparé au taux brut de mortalité par suicide observé parmi les hommes âgés de 15 à 59 ans de la population générale de l'hexagone (qui atteint 2,7 pour 10000 en 2010) ${ }^{(9)}$. En dehors du début des années 1860 et de la période qui suit la seconde guerre mondiale, le taux de suicide est toujours plus élevé en prison qu'en population

(8) À l'exception de la dernière période qui est de six ans.

(9) Avant 1925, les statistiques de décès par cause en population générale ne permettent pas de distinguer les 15-59 ans. Pour les périodes antérieures à 1925, le taux de suicide reporté figure 2 est celui de la population masculine tous âges publié par Jean-Claude Chesnais en 1976 (tableau annexe A.1). Les deux taux donnent des niveaux très similaires. Par ailleurs, il est d'usage, pour une série temporelle, de présenter des taux standardisés par âge mais les tendances du taux brut et du taux standardisé sont également très similaires (annexe A.3). 
Figure 2. Taux de suicide en prison et en population générale masculine en France métropolitaine depuis le milieu du XIX ${ }^{\mathrm{e}}$ siècle, (échelle logarithmique)

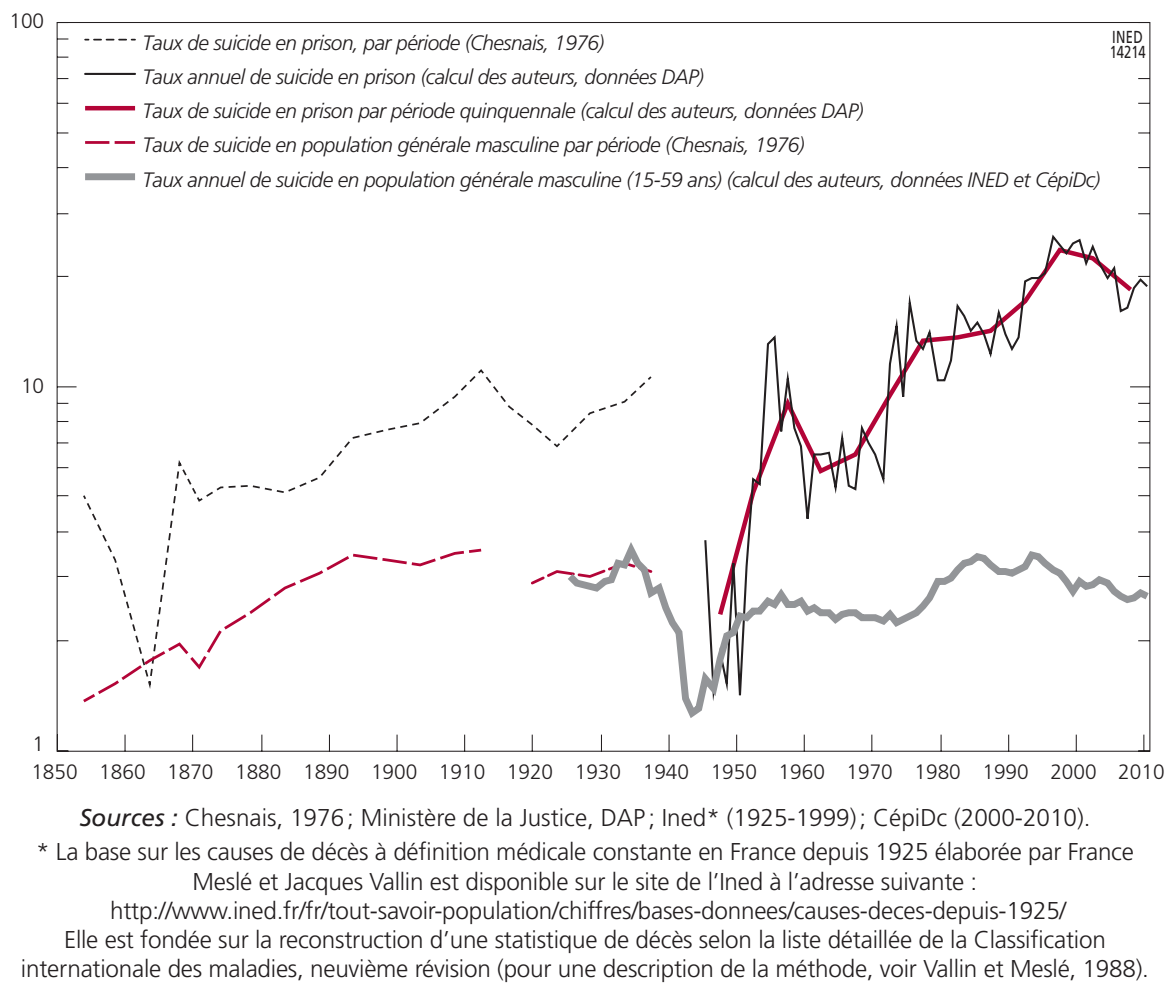

générale. Il est intéressant de noter que les tendances pour la population générale ne sont pas nécessairement les mêmes que celles observées en milieu carcéral (figure 2). En milieu libre, le niveau de suicide augmente à la fin des Trente Glorieuses et durant la crise économique qui lui succède (Chesnais et Vallin, 1981), mais ce lien s'est apparemment atténué depuis, et le niveau du suicide semble moins sensible à la crise économique de 2008 (Laanani et al., 2013).

Lévolution de la sursuicidité en prison par rapport au milieu libre peut être observée en calculant le rapport entre ces deux taux (figure 3). Alors que ce rapport oscille au-dessous de 4 avant la seconde guerre mondiale (4 fois plus de suicides en milieu carcéral qu'en milieu libre), il fluctue entre 6 et 9 au début des années 2000. Sur la période 2005-2010, le taux de suicide en prison est sept fois supérieur à celui observé en population générale. Ce rapport a bien sûr ses limites, la structure par sexe et par âge n'est pas identique et il existe une marge d'erreur dans l'estimation des taux de suicide, que ce soit en population générale ou en milieu carcéral. En population générale, la difficulté de certifier l'intentionnalité d'une mort violente (c'est-à-dire de pouvoir distinguer un suicide d'un accident ou d'un homicide) amène à une sous-évaluation de la mortalité par suicide : cette sous-estimation est actuellement estimée à $10 \%$ 
Figure 3. Sursuicidité de la population écrouée par rapport à la population générale masculine en France métropolitaine depuis le milieu du XIX ${ }^{e}$ siècle

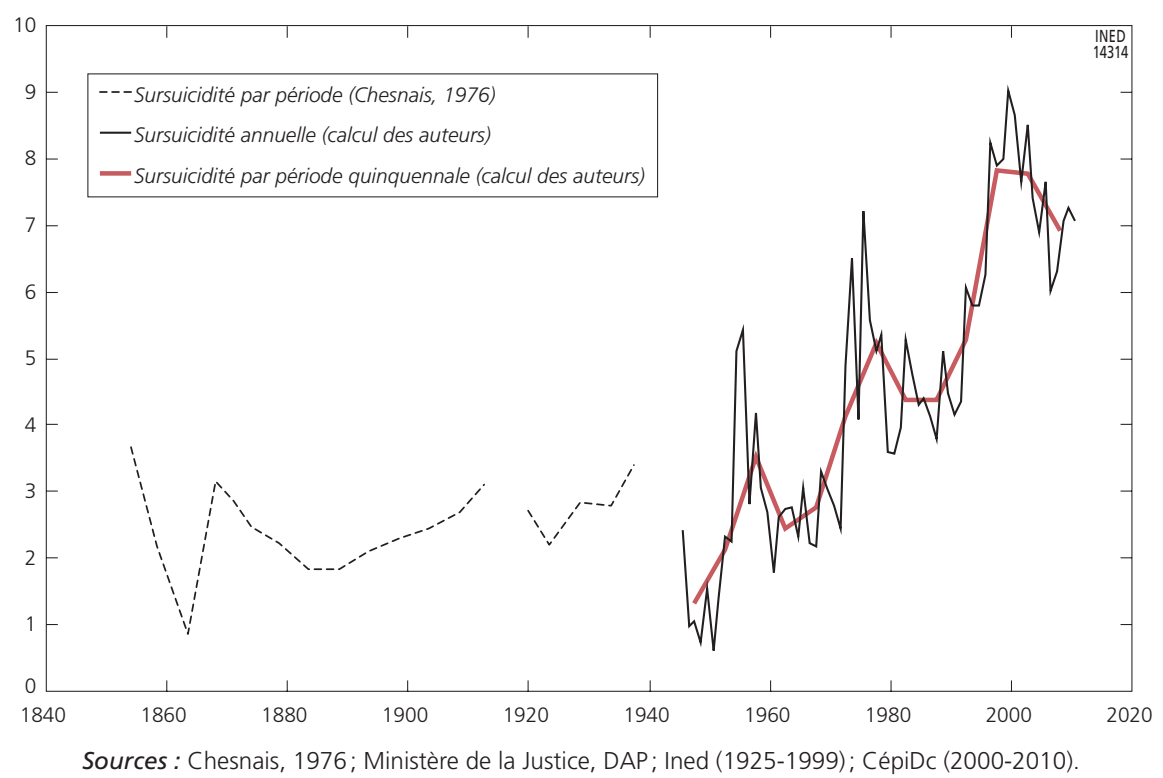

(Aouba et al., 2011). Le calcul de cette marge d'erreur n'a pas encore fait l'objet d'une étude spécifique en milieu carcéral. Toutefois, on peut supposer que la fiabilité des données a progressé au cours du temps dans les deux populations et que la prise en compte de cette marge d'erreur ne remettrait sans doute pas en question la conclusion d'une sursuicidité croissante en milieu carcéral par rapport au milieu libre.

Ne disposant pas de données temporelles permettant d'analyser précisément les raisons de la hausse du suicide en prison et de l'écart avec le milieu libre, il est difficile d'expliquer cette tendance. Au niveau des établissements, la surdensité carcérale détériore indéniablement les conditions de vie des détenus, ainsi que les relations qu'ils entretiennent avec le personnel surveillant et médico-social. Pourtant, Nicolas Bourgoin avait montré que la surpopulation carcérale et le suicide évoluait de façon inverse (1994), ce que l'on observe toujours aujourd'hui (Duthé et al., 2009, 2010). Ce contexte défavorable est en effet contrebalancé par un effet protecteur au niveau individuel puisque la présence d'un codétenu en cellule diminue le risque de suicide, soit parce qu'il diminue le sentiment d'isolement, soit parce qu'il empêche concrètement le détenu de passer à l'acte. Du point de vue démographique, les évolutions de la structure de population par sexe et âge (masculinisation de la population dans les années 1940-1950 et vieillissement dans les dernières décennies) sont relatives et n'ont pu qu'avoir un effet très marginal sur la différenciation (Duthé et al., 2010, 2011). L'hypothèse d'une augmentation du nombre de détenus atteints de troubles ou de pathologies mentales est parfois mise en avant mais 
difficilement vérifiable, ainsi que nous l'avons déjà mentionné. La mise en évidence de facteurs de risque spécifiques à la population carcérale (infraction motivant la mise sous écrou, incarcération, condamnation, conditions de vie et événements durant l'incarcération...), permet néanmoins d'apporter des éléments de réflexion.

\section{Les facteurs de risque du suicide en prison dans la période récente}

\section{Données, population et méthode}

Par le biais d'une convention établie entre la DAP et l'Ined respectant les règles éthiques en matière de confidentialité et de préservation des données personnelles, l'administration pénitentiaire nous a fourni des données provenant de la base nationale de Gestion informatisée des détenus en établissement (Gide) de l'administration pénitentiaire. La base Gide est une application informatique de gestion des personnes écrouées l'année de sa consultation ainsi que des trois dernières années civiles complètes. Ces informations portent sur les séjours sous écrou qui ont pu être observés en France entre le $1^{\mathrm{er}}$ janvier 2006 et le 15 juillet 2009. On dispose ainsi d'une période d'observation couvrant l'ensemble des séjours sous écrou, quelle que soit la date initiale de la mise sous écrou (qui peut être antérieure au $1^{\text {er }}$ janvier 2006). Durant cette période, 377688 séjours ont été dénombrés parmi lesquels 378 se sont terminés par le suicide de la personne écrouée. Le niveau d'observation est le séjour mais il est assimilé par simplification à la personne sous écrou, nous reviendrons sur ce point dans la discussion.

À partir des informations communiquées, nous avons constitué une base regroupant des données sur les caractéristiques de la personne écrouée susceptibles d'être associées au risque de suicide, eu égard aux facteurs mis en avant par d'autres études sur ce thème et rappelés en introduction. Ces caractéristiques sont de nature démographique (sexe, date de naissance, nationalité), pénale (date de mise sous écrou, date et raison de la levée de l'écrou, procédure pénale, infraction(s) motivant la mise sous écrou, date(s) de condamnation et sentence(s) prononcée(s) pour les personnes condamnées) et portent sur les conditions du séjour sous écrou (incarcération en établissement, type d'établissement pénitentiaire, présence d'un codétenu dans la cellule) ou sur les événements survenus durant la période d'observation (hospitalisations, fautes et sanctions disciplinaires dont le placement en cellule disciplinaire, visites au parloir). Dans la mesure où le nombre de suicides sur lequel porte l'analyse est relativement peu élevé par rapport à la population observée (environ 1 suicide sur 1000 séjours quelle que soit leur durée), nous observons un phénomène que l'on peut qualifier de statistiquement rare. Ainsi, une proportion non négligeable de données manquantes peut fausser les résultats et aboutir à des 
erreurs d'interprétation. C'est pourquoi certaines variables ont été retirées de l'analyse (maîtrise du français, niveau d'instruction, situation professionnelle et familiale, département du domicile). Déclarées par la personne au greffe au moment de sa mise sous écrou, elles sont d'une fiabilité incertaine et pas toujours bien saisies. En outre, en cas de transfert, ces informations peuvent avoir été mises à jour sans que l'on ait trace de cette éventuelle modification.

Une première étape d'analyse a consisté à modéliser le risque de suicide au moyen d'une régression logistique en tenant compte de l'ensemble des variables disponibles et exploitables. Des comparaisons ont été menées avec d'autres modèles supposés mieux adaptés pour l'observation d'un phénomène statistique rare (la régression de Poisson et la régression négative binomiale, cf. Orro et Senghor, 2010), mais les résultats se sont avérés identiques. Les résultats de cette première analyse ont été publiés par ailleurs (Duthé et al., 2010). Cependant, les données disponibles, notamment les dates des différents événements qui caractérisent le séjour, permettent de prendre en compte la durée d'exposition au risque, ce qui n'était pas le cas dans ce premier travail. La date de mise sous écrou et la date de fin d'écrou permettent en effet de construire un modèle de durée et de calculer les risques instantanés de se suicider en tenant compte des séjours qui peuvent être censurés à gauche (si la date de mise sous écrou est antérieure au $1^{\mathrm{er}}$ janvier 2006) et/ou à droite (si l'individu était toujours sous écrou au 15 juillet 2009).

Les variables indépendantes retenues ${ }^{(10)}$ sont d'ordre sociodémographique (sexe, âge à l'écrou, nationalité française) ou pénal (nature de l'infraction principale $^{(11)}$, situation pénale de la personne qui peut changer sur la période selon la date finale de condamnation dans le cas d'une condamnation, la durée de la peine de prison ferme prononcée le cas échéant); elles concernent les conditions de la mise sous écrou (incarcéré ou non) ou de détention (placement ou non en cellule disciplinaire durant la période à partir des dates éventuelles de début et de fin de placement). S'y ajoutent le fait d'avoir été hospitalisé (suivi d'un retour en cellule) durant la période observée ainsi que les visites d'un proche (parent ou ami). Pour cette analyse, nous avons écarté les séjours pour lesquels ces informations étaient manquantes ou incohérentes : des séjours pour lesquels l'individu restait plus de 5 ans sous écrou sans avoir été condamné d'après les données de la base (moins de 0,1 \% des séjours), d'autres pour lesquels l'âge à l'écrou était inconnu (0,6\%) ou aucune information n'était

\footnotetext{
(10) Ne disposant pas de l'information sur l'ensemble des séjours, nous avons écarté deux caractéristiques d'intérêt : le type d'établissement et le nombre de codétenus. Ces deux caractéristiques, connues à la fin de l'observation, sont associées au risque de se suicider : ce dernier est plus élevé parmi les personnes placées en maison d'arrêt (ou en quartier maison d'arrêt au sein d'autres établissements) que parmi les individus placés en établissement pour peine. Le risque est aussi plus élevé pour les personnes seules en cellule (Duthé et al., 2010).

(11) Les infractions ont été hiérarchisées en six catégories: (1) meurtre; (2) viol; (3) autre atteinte aux mœurs; (4) violence volontaire; (5) autre infraction (vol aggravé, délit et vol simple, infraction à la législation sur les stupéfiants, autre délit). En cas de cumul d'infractions, la première sur cette échelle a été retenue.
} 
disponible sur les infractions (2,6\%). L'analyse a finalement porté sur 363525 séjours et 377 suicides.

Pour décrire la population étudiée, nous présentons les durées moyennes passées sous écrou et les durées de séjour observées dans cette étude. En effet, les différences de durée passée sous écrou selon les caractéristiques de la personne entraînent des différences de durée observée sur la période, et jouent sur le nombre de personne-années observées pour les différents profils de personnes écrouées qui permettent le calcul d'un taux précis de suicide en prison. Nous présentons ensuite la fonction de survie associée au risque de suicide sous écrou. Pour finir, nous étudions les différences de niveaux de mortalité par suicide selon le profil des personnes et séjours sous écrou, en calculant des taux de mortalité puis par une analyse multivariée.

Pour l'analyse multivariée, nous utilisons le modèle des risques proportionnels de Cox. Ce modèle mesure la différence sur le risque instantané (hazard) de connaître l'événement considéré selon une caractéristique en calculant rapport à une autre. Il modélise les quotients instantanés de la façon suivante :

$$
h(t, z)=h_{0}(t) \cdot \exp (\beta \cdot z)
$$

où z caractérise l'individu (ici le séjour) selon l'ensemble des caractéristiques retenues $\left(z_{1}, z_{2}, z_{3} \ldots\right), \mathrm{h}_{0}(\mathrm{t})$ est le quotient instantané (ou le risque) de connaître l'événement pour l'individu de référence dont toutes les caractéristiques sont nulles $(z=0)$, à la durée $t$. Ce modèle ne décrit pas l'évolution dans la durée des risques de connaître l'événement selon les différentes caractéristiques individuelles, mais il mesure le rapport qui existe entre ces risques, rapport que l'on suppose constant dans la durée :

$$
\frac{h(t, z)}{h_{0}(t)}=\exp (\beta \cdot z)
$$

Il repose donc sur l'hypothèse des risques proportionnels dont il faut s'assurer de la validité. Des tests permettent de tester la spécificité du modèle. Dans cette analyse, nous avons utilisé la méthode des résidus de Schoenfeld (Hosmer et al., 2008).

\section{Durées de séjours sous écrou}

Les séjours passés sous écrou sont pour la plupart de durées relativement courtes. La moitié des séjours sous écrou qui se sont terminés entre le $1^{\text {er }}$ janvier 2006 et le 15 juillet 2009 ont duré moins de 0,34 an soit quatre mois, et la durée moyenne était de 0,72 an (un peu moins de neuf mois). L'ensemble des séjours observés sur cette même période ont été d'une durée moyenne de 0,61 an soit un peu plus de sept mois (tableau 1).

La durée des séjours varie selon les caractéristiques : elle est généralement plus courte pour les femmes, les mineurs au moment de la mise sous écrou, les personnes sous écrou mais non incarcérées, les prévenus, les personnes 
Tableau 1. Durée des séjours sous écrou entre le 01/01/2006 et le 15/07/2009, selon différentes caractéristiques (années)

\begin{tabular}{|c|c|c|c|}
\hline & $\begin{array}{l}\text { Durée médiane } \\
\text { de séjour }{ }^{(a)}\end{array}$ & $\begin{array}{l}\text { Durée moyenne } \\
\text { de séjour }{ }^{(\mathrm{a})}\end{array}$ & $\begin{array}{l}\text { Durée moyenne } \\
\text { observé } e^{(b)}\end{array}$ \\
\hline Ensemble & 0,34 & $0,72(0,72-0,73)$ & $0,61(0,61-0,61)$ \\
\hline Homme & 0,34 & $0,73(0,72-0,73)$ & $0,61(0,61-0,62)$ \\
\hline Femme & 0,28 & $0,59(0,57-0,61)$ & $0,52(0,51-0,53)$ \\
\hline Mineur à l'écrou & 0,15 & $0,36(0,34-0,37)$ & $0,32(0,31-0,33)$ \\
\hline Âgé de 18 à moins de 30 ans à l'écrou & 0,37 & $0,68(0,68-0,69)$ & $0,59(0,59-0,60)$ \\
\hline Âgé de 30 ans ou plus à l'écrou & 0,31 & $0,79(0,78-0,80)$ & $0,65(0,64-0,65)$ \\
\hline Français & 0,34 & $0,73(0,72-0,73)$ & $0,61(0,61-0,62)$ \\
\hline Étranger & 0,32 & $0,70(0,69-0,71)$ & $0,59(0,59-0,60)$ \\
\hline Hébergé en établissement & 0,35 & $0,75(0,74-0,76)$ & $0,63(0,63-0,63)$ \\
\hline Non hébergé & 0,25 & $0,39(0,39-0,40)$ & $0,35(0,34-0,35)$ \\
\hline Prévenu ${ }^{(c)}$ & 0,24 & $0,33(0,33-0,33)$ & $0,31(0,30-0,31)$ \\
\hline Condamné(c) & 0,37 & $0,81(0,81-0,82)$ & $0,60(0,60-0,61)$ \\
\hline Condamné à moins de 1 an ferme ${ }^{(c)}$ & 0,20 & $0,30(0,30-0,30)$ & $0,27(0,27-0,27)$ \\
\hline $\begin{array}{l}\text { Condamné de } 1 \text { à moins de } 10 \text { ans } \\
\text { fermes }\end{array}$ & 0,99 & $1,79(1,78-1,80)$ & $1,11(1,11-1,12)$ \\
\hline Condamné à 10 ans fermes ou plus(c) & 5,59 & $9,24(9,14-9,35)$ & $2,59(2,57-2,61)$ \\
\hline Écroué pour meurtre & 2,39 & $4,07(3,93-4,22)$ & $1,94(1,92-1,97)$ \\
\hline Écroué pour viol & 2,09 & $3,63(3,55-3,71)$ & $1,75(1,73-1,77)$ \\
\hline Écroué pour une autre agression sexuelle & 0,59 & $1,03(1,00-1,05)$ & $0,78(0,77-0,79)$ \\
\hline Écroué pour violence volontaire & 0,38 & $0,69(0,68-0,69)$ & $0,62(0,62-0,63)$ \\
\hline Écroué pour une autre infraction & 0,29 & $0,53(0,53-0,53)$ & $0,47(0,47-0,47)$ \\
\hline \multicolumn{4}{|c|}{ 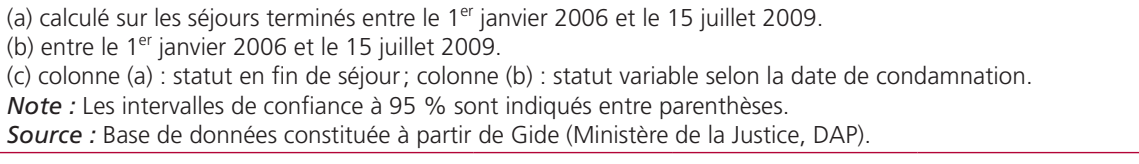 } \\
\hline
\end{tabular}

condamnées à des peines de prison ferme inférieures à un an, et les personnes écrouées pour une infraction autre que meurtre, viol, agression sexuelle ou violence volontaire. Les personnes écrouées pour meurtre, viol ou autre agression sexuelle ont en moyenne respectivement passé 4,1 ans, 3,6 ans et 1,0 an sous écrou.

\section{Le suicide, un phénomène statistiquement rare}

La fonction de survie associée au modèle de durée représente la probabilité cumulée de ne pas se suicider au cours du temps, depuis la mise sous écrou (figure 4). Cette probabilité reste relativement élevée dans les premières années : après 10 ans passés sous écrou, la probabilité cumulée de ne pas se suicider est de 0,9847 (95\% IC =0,9810 - 0,9876). Le suicide en prison est bien un phénomène statistiquement rare. Au-delà de 15 ans sous écrou, l'intervalle de confiance s'élargit et les effectifs de séjours s'amenuisent, ce qui ne permet pas une bonne estimation des risques instantanés de suicide. 
Figure 4. Fonction de survie (estimation et intervalle de confiance à $95 \%$ ) du risque de suicide en prison depuis le début de la mise sous écrou (années)

4A. Ensemble des séjours observés

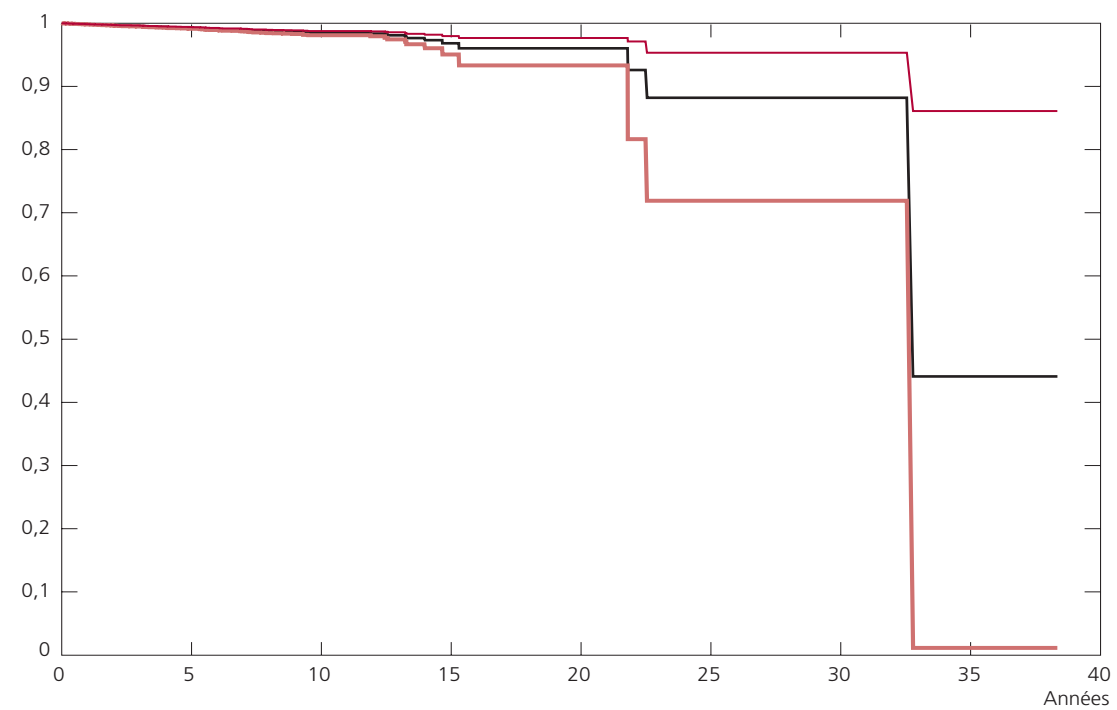

4B. Les 10 premières années

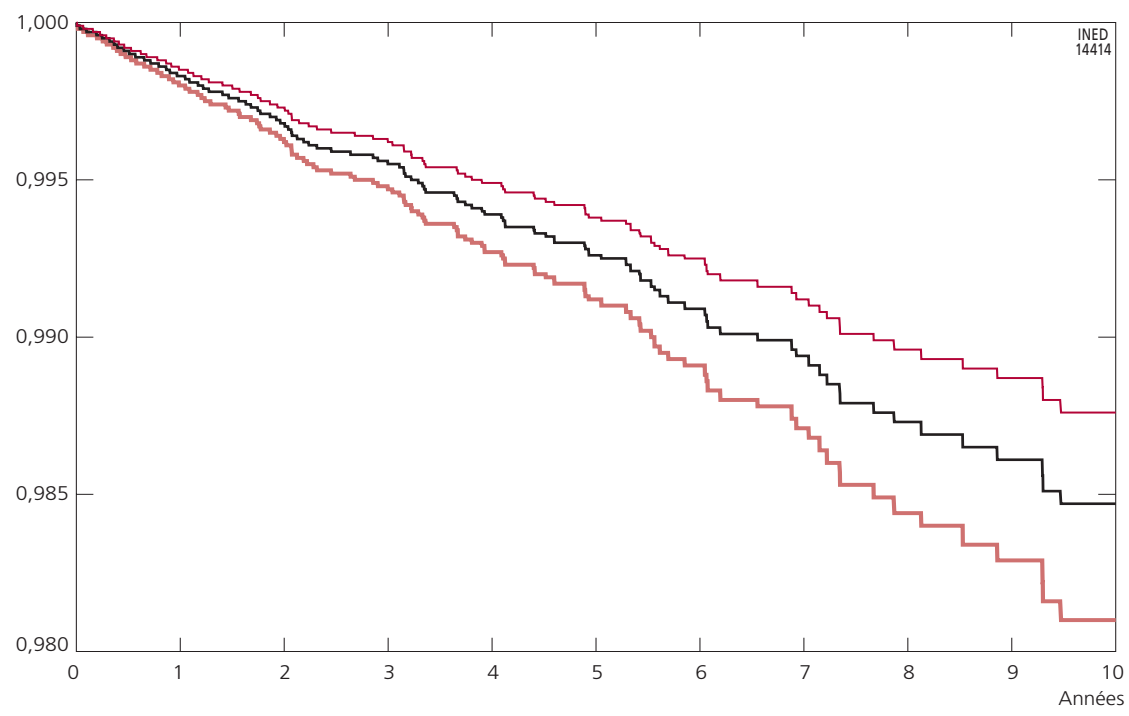

Source : Base de données constituée à partir de Gide (Ministère de la Justice, DAP).

\section{Taux de mortalité par suicide en prison}

De 2006 à mi-2009, le taux de mortalité par suicide était de 17 suicides pour 10000 personnes écrouées sur une année (95\% IC = 15,4-18,8) (tableau 2). Pour cette même période, le taux brut calculé sur la population moyenne est 
Tableau 2. Personnes-années, suicides et taux de mortalité par suicide (pour 10000) pour l'ensemble des séjours observés

du 01/01/2006 au 15/07/2009 en France, selon différentes caractéristiques

\begin{tabular}{|c|c|c|c|}
\hline & $\begin{array}{l}\text { Personnes- } \\
\text { années }\end{array}$ & $\begin{array}{l}\text { Nombre de } \\
\text { suicides }\end{array}$ & $\begin{array}{l}\text { Taux de mortalité } \\
\text { par suicide }\end{array}$ \\
\hline Ensemble & 221348,3 & 377 & $17,0(15,4-18,8)$ \\
\hline Homme & 213256,2 & 366 & $17,2(15,5-19,0)$ \\
\hline Femme & 8092,1 & 11 & $13,6(7,5-24,5)$ \\
\hline Mineur à l'écrou & 3953,9 & 4 & $10,1(3,8-27,0)$ \\
\hline Âgé de 18 à moins de 30 ans à l'écrou & 138994,0 & 203 & $14,6(12,7-16,8)$ \\
\hline Âgé de 30 ans ou plus à l'écrou & 78400,5 & 170 & $21,7(18,7-25,2)$ \\
\hline Français & 178563,7 & 307 & $17,2(15,4-19,2)$ \\
\hline Étranger & 42784,6 & 70 & $16,4(12,9-20,7)$ \\
\hline Hébergé en établissement & 211418,7 & 368 & $17,4(15,7-19,3)$ \\
\hline Non hébergé & 9929,6 & 9 & $9,1(4,7-17,4)$ \\
\hline En cellule ordinaire & 219651,1 & 347 & $15,8(14,2-17,6)$ \\
\hline En cellule disciplinaire & 1697,2 & 30 & $176,8(123,6-252,8)$ \\
\hline Pas d'hospitalisation durant la période & 170729,5 & 246 & $14,4(12,7-16,3)$ \\
\hline Hospitalisé durant la période & 50618,9 & 131 & $25,9(21,8-30,7)$ \\
\hline Pas de visite de proche durant la période & 134426,2 & 293 & $21,8(19,4-24,4)$ \\
\hline Au moins une visite durant la période & 86922,2 & 84 & $9,7(7,8-12,0)$ \\
\hline Prévenu & 44485,1 & 151 & $33,9(28,9-39,8)$ \\
\hline Condamné & 176863,2 & 226 & $12,8(11,2-14,6)$ \\
\hline Condamné à moins de 1 an ferme & 53633,3 & 69 & $12,9(10,2-16,3)$ \\
\hline $\begin{array}{l}\text { Condamné de } 1 \text { à moins de } 10 \text { ans } \\
\text { fermes }\end{array}$ & 93474,0 & 97 & $10,4(8,5-12,7)$ \\
\hline Condamné à 10 ans fermes ou plus & 29755,9 & 60 & $20,2(15,7-26,0)$ \\
\hline Écroué pour meurtre & 17875,2 & 85 & $47,6(38,4-58,8)$ \\
\hline Écroué pour viol & 28292,2 & 77 & $27,2(21,8-34,0)$ \\
\hline Écroué pour autre agression sexuelle & 10023,0 & 24 & $23,9(16,1-35,7)$ \\
\hline Écroué pour violence volontaire & 49491,4 & 80 & $16,2(13,0-20,1)$ \\
\hline Écroué pour une autre infraction & 115666,6 & 111 & $9,6(8,0-11,6)$ \\
\hline
\end{tabular}

de 17,4 : ce taux brut aboutit donc à une légère surestimation mais l'ordre de grandeur reste le même.

Du point de vue des caractéristiques sociodémographiques, malgré un taux plus faible pour les femmes $(13,6)$ que pour les hommes $(17,2)$, la différence n'est pas significative, notamment en raison du faible effectif de femmes. Il en va de même pour les mineurs qui enregistrent un taux de 10,1 pour 10000 . Les étrangers enregistrent un taux un peu plus faible $(16,4)$ que les Français 
$(17,2)$ mais cette différence n'est pas significative. Par contre, les adultes écroués après l'âge de 30 ans ont un taux significativement plus élevé que les plus jeunes adultes au moment de l'écrou $(21,7$ contre 14,6$)$.

Du point de vue des conditions d'incarcération, les personnes non détenues enregistrent un taux de mortalité par suicide de 9,1 pour 10000 , ce qui est bien moins élevé que celles qui résident en établissement, mais l'intervalle de confiance est large et cette différence n'est pas significative. En revanche, même si l'on tient compte de l'intervalle de confiance, on observe un taux de suicide extrêmement élevé lorsque le détenu est placé en cellule disciplinaire (176,8 pour $10000,95 \%$ IC $=123,6-252,8$ ). Les personnes hospitalisées au moins une fois sur la période observée ont un taux plus élevé que les autres (25,9 contre 14,4$)$ et il en est de même pour les personnes sous écrou qui n'ont reçu aucune visite de leur proche durant cette période (21,8 contre 9,7).

S'agissant du profil pénal, les prévenus ont un taux de mortalité par suicide près de trois fois plus élevé que les condamnés $(33,9$ contre 12,8$)$ et parmi ces derniers, ceux condamnés à plus de 10 ans fermes ont un taux plus élevé que les autres $(20,2)$. Le taux de suicide varie selon la nature de linfraction principale : il est le plus élevé parmi les personnes écrouées pour meurtre (47,6 pour 10000), il est de 27,2 pour viol, 23,9 pour une autre agression sexuelle, 16,2 pour une violence volontaire et de 9,6 pour une autre infraction.

Pour résumer, le taux de suicide est particulièrement élevé pour les individus placés en cellule disciplinaire, les personnes écrouées pour des infractions graves, prévenues ou condamnées à de longues peines, les individus ayant vécu récemment une hospitalisation, ayant déjà atteint un certain âge au moment de la mise sous écrou. À l'inverse, les mineurs à l'écrou, les personnes écrouées sans être incarcérées ou encore celles recevant des visites de proches présentent des taux relativement bas (autour de 10 pour 10 000). Dans la partie suivante, l'analyse multivariée effectuée permet de vérifier (ou non) la persistance de ces associations en tenant compte de l'ensemble des caractéristiques de manière simultanée.

\section{Des caractéristiques sociodémographiques peu significatives?}

Dans cette partie et les suivantes, nous interprétons les résultats de l'analyse multivariée présentés dans le tableau 3. Toutes choses égales par ailleurs et sous l'hypothèse que le rapport des risques est constant dans la durée, les risques ne sont pas significativement différents selon le sexe, l'âge à l'écrou et la nationalité. Les rapports de risque montrent des risques moins élevés pour les femmes et les mineurs, mais les intervalles de confiance sont trop larges pour pouvoir vérifier une association statistique. Il est fort probable que les faibles effectifs associés à ces catégories soient la cause de cette non-significativité. Lâge â l'écrou qui paraissait significatif dans l'analyse bivariée ne l'est qu'à $7 \%$ et l'hypothèse des risques proportionnels n'est pas vérifiée. Une analyse restreinte aux hommes adultes incarcérés montre un effet significatif de l'âge à 
Tableau 3. Rapport du risque instantané de suicide (modèle de Cox) pour l'ensemble des séjours observés du 01/01/2006 au 15/07/2009 en France, selon différentes caractéristiques

\begin{tabular}{|c|c|c|}
\hline & Rapport de risque & $P>z$ \\
\hline Homme (Réf.) & 1 & \\
\hline Femme & $0,6(0,3-1,2)$ & 0,194 \\
\hline Mineur à l'écrou & $0,4(0,2-1,2)$ & 0,110 \\
\hline Âgé de18 à moins de 30 ans à l'écrou (Réf.) & 1 & \\
\hline Âgé de 30 ans ou plus à l'écrou & $1,2(1-1,5)$ & $0,071(\mathrm{np})$ \\
\hline Français (Réf.) & 1 & \\
\hline Étranger & $0,9(0,7-1,1)$ & 0,345 \\
\hline Hébergé en établissement (Réf.) & 1 & \\
\hline Non hébergé & $0,7(0,3-1,3)$ & 0,232 \\
\hline En cellule ordinaire (Réf.) & 1 & \\
\hline En cellule disciplinaire & $15,4(10,5-22,6)$ & 0,000 \\
\hline Pas d'hospitalisation durant la période (Réf.) & 1 & \\
\hline Hospitalise durant la période & $1,7(1,3-2,1)$ & 0,000 \\
\hline Pas de visite de proche durant la période (Réf.) & 1 & \\
\hline Au moins une visite durant la période & $0,4(0,3-0,5)$ & 0,000 \\
\hline Prévenu & $1,9(1,4-2,6)$ & 0,000 \\
\hline Condamné à moins de 1 an de prison ferme (Réf.) & 1 & \\
\hline Condamné de 1 à moins de 10 ans fermes & $0,9(0,6-1,3)$ & 0,656 \\
\hline Condamné à 10 ans fermes ou plus & $1,2(0,7-2,1)$ & 0,557 \\
\hline Écroué pour meurtre & $7,3(5,2-10,2)$ & 0,000 \\
\hline Écroué pour viol & $4,2(3,0-5,9)$ & 0,000 \\
\hline Écroué pour autre agression sexuelle & $2,7(1,7-4,2)$ & 0,000 \\
\hline Écroué pour violence volontaire & $1,9(1,4-2,5)$ & 0,000 \\
\hline Écroué pour une autre infraction (Réf.) & 1 & \\
\hline \multicolumn{3}{|c|}{$\begin{array}{l}\text { (np) ne répond pas à l'hypothèse de proportionnalité. } \\
\text { Note : Les intervalles de confiance à } 95 \% \text { sont indiqués entre parenthèses. } \\
\text { Source : Base de données constituée à partir de Gide (Ministère de la Justice, DAP). }\end{array}$} \\
\hline
\end{tabular}

l'écrou avec un sur-risque pour les plus âgés (Duthé et al., 2013). Nos observations diffèrent de celles effectuées dans d'autres pays, notamment au RoyaumeUni où ce sont les adultes les plus jeunes qui paraissent les plus vulnérables (Humber et al., 2011). Cependant, aux États-Unis, les études ne s'accordent pas nécessairement sur l'effet de l'âge (Way et al., 2005). Ces variations d'un contexte et d'une étude à l'autre font que l'âge n'est pas clairement reconnu comme un facteur de risque (Fazel et al., 2008). En outre, notre analyse se singularise des autres du fait que nous étudions l'effet de l'âge à l'écrou et non de lâge au suicide. Pour le modèle de durée constitué ici, notre intérêt était de considérer la durée depuis la mise sous écrou plutôt que lâge au suicide. Par ailleurs, nous disposons de peu de données sociodémographiques comme le statut marital, le fait d'avoir des enfants, etc., pour les raisons expliquées précédemment, il est donc difficile de conclure sur le faible rôle des variables sociodémographiques dans le suicide en prison. 


\section{Hospitalisation et état de santé des personnes sous écrou}

Les données sanitaires des personnes sous écrou relèvent du ministère de la Santé et n'étaient pas disponibles pour cette étude. Par ailleurs, l'administration pénitentiaire n'enregistre pas de manière systématique les tentatives de suicide, sauf si elles ont engendré des incidents au sein de l'établissement. Les éventuels transferts d'un détenu vers une structure de soins durant la période observée constituent la seule information sanitaire dont nous disposons et nous la considérons ici comme une mesure approchée de l'état de santé de l'individu. Confirmant l'analyse bivariée, le risque de suicide est significativement plus élevé $(1,7)$ pour les personnes ayant été hospitalisées auparavant (hospitalisations suivies d'une réincarcération) que pour les autres. Il existe une très forte association entre le suicide et les troubles mentaux et du comportement, comme les problèmes d'addiction et les comportements suicidaires ${ }^{(12)}$. Il est possible que les motifs d'hospitalisation, non disponibles, soient liés dans une certaine mesure à un problème de santé mentale.

\section{Mise en isolement et visite des proches}

S'agissant des conditions de la mise sous écrou, le rapport des risques est moindre pour les personnes non incarcérées mais l'écart n'est pas significatif contrairement à l'analyse bivariée qui montrait une nette différence. Les faibles effectifs de personnes non incarcérées limitent probablement la portée des résultats. Par contre, et ce malgré de faibles effectifs, les moments passés en cellule disciplinaire sont considérablement plus à risques que ceux passés en cellule ordinaire (rapport du risque instantané de plus de 15). Le placement en cellule disciplinaire est, dans le vocabulaire pénitentiaire, une sanction donnée suite à une faute, qui a pu être relativement grave (troubles dans l'établissement, comportement violent à l'égard d'un détenu ou d'un surveillant...). Les personnes placées en cellule disciplinaire sont donc des personnes qui expriment une difficulté à s'adapter à l'environnement dans lequel elles évoluent (Liebling, 1995). Il est possible qu'elles soient également plus enclines à avoir des troubles du comportement et mentaux. Le rapport du risque est particulièrement élevé, ce qui montre bien que l'isolement physique est une situation particulièrement vulnérabilisante du point de vue du suicide.

Au-delà de l'isolement physique, notre étude révèle aussi l'importance du lien social entretenu entre le détenu et ses proches dans la mesure où le fait d'avoir reçu au moins une visite d'un parent ou d'un ami amoindrit le risque de suicide $(0,4)$. Ces résultats confirment le rôle du soutien social mis en avant dans des études plus qualitatives, notamment au Royaume-Uni auprès de femmes (Marzano et al., 2011) et de jeunes (Liebling, 1993) en prison. Par ailleurs, les personnes atteintes de troubles mentaux et du comportement sont socialement plus isolées que les autres, ce qui peut renforcer l'association entre l'absence de visite et le risque suicidaire.

(12) Liste de références dans la note de bas de page $n^{\circ} 4$. 


\section{L'infraction principale ayant motivé la mise sous écrou}

Dans le modèle multivarié, on retrouve de manière significative et prononcée le gradient du risque de suicide selon la nature de l'infraction principale qui a motivé la mise sous écrou. Le rapport de risques est de 7,3 pour un meurtre, 4,2 pour un viol, 2,7 pour une autre agression sexuelle, 1,9 pour une violence volontaire, par rapport aux autres infractions. Plusieurs facteurs peuvent expliquer le lien entre suicide et gravité de l'infraction principale : l'infraction en elle-même, les sentiments de remords par rapport à l'acte commis ou le sentiment d'injustice suite à la mise sous écrou; le stress et l'incertitude quant à la condamnation avant le procès; le verdict de culpabilité et une lourde peine de prison une fois la condamnation prononcée. Ainsi, la durée de la peine est un facteur secondaire, ce qui expliquerait qu'il n'y a pas de différence selon la longueur de la peine, une fois que l'on contrôle la nature de l'infraction, contrairement à ce qu'on pouvait observer dans l'analyse bivariée.

En ce qui concerne le rôle majeur du lien social, certaines infractions, en particulier le viol, peuvent être à l'origine d'ostracisme au sein même de la prison (Harvey et Liebling, 2001). On sait aussi que dans la grande majorité des violences envers les personnes, l'auteur et sa victime se connaissent. Beaucoup de violences, notamment des meurtres et des viols, sont commis dans l'entourage et même dans la famille (Mucchielli, 2002; Bajos et Bozon, 2008), la rupture avec les proches est alors d'autant plus probable.

\section{La période de détention provisoire}

Du point de vue des caractéristiques pénales, si la longueur de la peine n'apparaît plus significative dans le modèle global, les prévenus se suicident deux fois plus que les condamnés. Or, la période de détention provisoire est vécue par la plupart des individus au moment de leur mise sous écrou, sauf dans le cas de condamnations immédiates. Cette mise sous écrou, qui se traduit la plus grande partie du temps par une incarcération, génère un choc violent pour la personne qui passe du milieu libre à la prison (Terra, 2003; OMS, 2007). Cette incarcération marque la rupture avec les proches, la restriction des libertés, la nécessité de s'adapter à ce nouvel environnement et, pour beaucoup, une absence de perspective d'avenir. Nous l'avons évoqué dans le cas des infractions graves, mais l'incertitude quant à la condamnation et la peine prononcée reste aussi une donnée stressante pour l'ensemble des personnes en détention provisoire.

\section{Conclusion}

Dans cette étude, nous ne disposons pas d'information sur l'état de santé des personnes mises sous écrou, à l'exception des hospitalisations sur la période d'observation. Les événements survenus depuis l'incarcération jusqu'au moment 
de l'observation pour les séjours déjà commencés au $1^{\mathrm{er}}$ janvier ne sont pas connus. Nous n'avons pas non plus d'éléments biographiques sur la personne avant son incarcération (antécédents judiciaires, événements traumatisants, comportements suicidaires...) qui pourraient apporter d'autres éléments sur les causes de suicide (Liebling, 1995). Le comportement suicidaire peut être préalable à l'incarcération, comme cela a été observé pour des détenus ayant commis un crime contre un proche (Bourgoin, 1993c). En outre, l'unité d'analyse étant basée sur le séjour en prison et non sur la personne, nous ne pouvons pas identifier si une seule personne a effectué plusieurs séjours pendant la période observée. Pour les récidivistes, le choc de l'incarcération peut être moindre, mais la peine (attendue ou prononcée) peut aussi être plus sévère. Toutefois, les récidivistes sont surtout des jeunes adultes incarcérés pour des délits (Kensey et Benaouda, 2011), moins vulnérables au suicide. Enfin, il ne nous a pas été possible d'étudier les éléments de contexte en intégrant les caractéristiques des établissements (surpopulation, formation des surveillants, présence d'une structure sanitaire...) dans l'analyse, faute d'effectifs suffisamment importants pour mener une analyse multiniveau. Nous n'avons pas non plus appréhendé les événements à venir tels que la libération qui peut être génératrice d'un grand stress (Terra, 2003).

Dans la première partie de cet article, nous avons montré la spécificité croissante du suicide en milieu carcéral par rapport à la population générale. Il n'a pas été possible de mettre en avant des corrélations claires avec la situation sociale et économique, ce qui limite la comparaison avec les facteurs de risque en milieu libre, mais les résultats présentés en deuxième partie confirment l'importance de caractéristiques pénales, notamment la nature des infractions commises, et des conditions de vie liées à la mise sous écrou et à l'incarcération. Des caractéristiques qui sont donc propres aux détenus. Une étude plus qualitative menée sur les dernières lettres des détenus s'étant suicidés souligne par ailleurs la diversité des situations et des tensions qui conduisent au suicide (Pan Ké Shon, 2013).

D'après le dernier plan d'actions de prévention et de lutte contre le suicide carcéral, de nouvelles mesures sont prévues pour diminuer le sentiment d'isolement ressenti par les détenus placés en cellule disciplinaire, par exemple en leur donnant accès à un téléphone ou une radio (Terra 2003, Albrand 2009). Par ailleurs, le passage brutal du milieu libre au milieu carcéral reste un choc au moment de l'incarcération. La prison marque une rupture forte dans la vie des personnes à tous les points de vue : perte des liens sociaux et familiaux, de liberté, de perspectives d'avenir, etc. La forte tension vécue par l'individu atteint son paroxysme dans l'isolement induit par son placement en cellule disciplinaire. Le taux de suicide y approche 2 \% une fois contrôlée la durée passée en cellule disciplinaire. La vigilance lors de la détention provisoire doit également rester maximale. Enfin, au-delà des conditions de détention, la nature de l'infraction doit aussi être prise en compte dans les actions à mener pour prévenir le suicide en prison. 
Ces dernières années ont été marquées par le développement d'aménagements de peine permettant de diminuer le temps passé en détention. Dans l'étude du suicide en prison, la distinction entre la population carcérale et la population écrouée mais non hébergée devient de plus en plus importante à faire. Dans cette perspective, il serait aussi intéressant d'étendre l'analyse du suicide à l'ensemble des personnes prises en charge par l'administration pénitentiaire. En effet, plus des deux tiers de ces personnes sont suivies par les services pénitentiaires d'insertion et de probation en milieu ouvert, soit indépendamment, soit consécutivement à une prise en charge sous écrou. Toutefois, la méthodologie reste à établir car il n'existe pas de signalement des suicides de personnes suivies en milieu ouvert.

Remerciements : Les auteures remercient les membres du Bureau des études et de la prospective, de la Mission de prévention et de lutte contre le suicide en milieu carcéral, du Bureau des systèmes d'information de la direction de l'administration pénitentiaire ainsi que le service Méthodes et statistiques de l'Ined. 
ANNEXES 
Tableau A.1. Évolution par période de la population moyenne sous écrou, du taux de suicide en prison et du taux de suicide en population générale masculine (p. 10000) du milieu du XIX ${ }^{e}$ siècle jusqu'à la veille de la seconde guerre mondiale en France métropolitaine

\begin{tabular}{|c|c|c|c|c|}
\hline Période & $\begin{array}{l}\text { Population moyenne } \\
\text { sous écrou } \\
\text { (1) }\end{array}$ & $\begin{array}{l}\text { Taux de suicide } \\
\text { en prison } \\
\text { (2) }\end{array}$ & $\begin{array}{l}\text { Taux de suicide } \\
\text { en population générale } \\
\text { Hommes } \\
\text { (3) }\end{array}$ & $\begin{array}{c}\text { Sursuicidité } \\
\text { des personnes sous } \\
\text { écrou } \\
\text { (2)/(3) }\end{array}$ \\
\hline $1852-1855$ & 25467 & 5,0 & 1,4 & 3,7 \\
\hline $1856-1860$ & 27426 & 3,3 & 1,5 & 2,2 \\
\hline 1861-1865 & 25023 & 1,5 & 1,8 & 0,9 \\
\hline 1866-1869 & 40644 & 6,2 & 2,0 & 3,2 \\
\hline 1870-1871 & 34091 & 4,9 & 1,7 & 2,9 \\
\hline $1872-1875$ & 43579 & 5,3 & 2,1 & 2,5 \\
\hline $1876-1880$ & 41996 & 5,3 & 2,4 & 2,2 \\
\hline 1881-1885 & 40141 & 5,1 & 2,8 & 1,8 \\
\hline $1886-1890$ & 37402 & 5,6 & 3,1 & 1,8 \\
\hline 1891-1895 & 34589 & 7,3 & 3,5 & 2,1 \\
\hline $1896-1900$ & 28008 & 7,6 & 3,3 & 2,3 \\
\hline 1901-1905 & 22520 & 7,9 & 3,2 & 2,5 \\
\hline 1906-1910 & 22003 & 9,4 & 3,5 & 2,7 \\
\hline 1911-1913 & 24195 & 11,1 & 3,6 & 3,1 \\
\hline 1914-1918 & 17614 & 8,9 & - & - \\
\hline 1919-1920 & 25481 & 7,9 & 2,9 & 2,7 \\
\hline 1921-1925 & 24152 & 6,9 & 3,1 & 2,2 \\
\hline 1926-1930 & 21505 & 8,5 & 3,0 & 2,8 \\
\hline 1931-1935 & 19121 & 9,1 & 3,3 & 2,8 \\
\hline 1936-1938 & 15073 & 10,6 & 3,1 & 3,4 \\
\hline
\end{tabular}


Tableau A.2. Population moyenne sous écrou, taux de suicide en prison et taux de suicide en population générale masculine de 15 à 59 ans (p. 10000), France métropolitaine, 1945-2010

\begin{tabular}{|c|c|c|c|c|}
\hline Période & $\begin{array}{l}\text { Population moyenne } \\
\text { sous écrou } \\
\text { (1) }\end{array}$ & $\begin{array}{l}\text { Taux de suicide } \\
\text { des personnes } \\
\text { sous écrou (2) }\end{array}$ & $\begin{array}{l}\text { Taux de suicide } \\
\text { en population générale } \\
\text { Hommes } 15-59 \text { ans (3) }\end{array}$ & $\begin{array}{c}\text { Sursuicidité des } \\
\text { personnes sous écrou } \\
(2) /(3)\end{array}$ \\
\hline 1945 & 63051 & 3,8 & 1,6 & 2,4 \\
\hline 1946 & 62210 & 1,4 & 1,5 & 1,0 \\
\hline 1947 & 59070 & 1,9 & 1,8 & 1,0 \\
\hline 1948 & 52552 & 1,5 & 2,1 & 0,7 \\
\hline 1949 & 42543 & 3,3 & 2,1 & 1,6 \\
\hline 1950 & 35257 & 1,4 & 2,4 & 0,6 \\
\hline 1951 & 31072 & 3,2 & 2,3 & 1,4 \\
\hline 1952 & 26802 & 5,6 & 2,4 & 2,3 \\
\hline 1953 & 23941 & 5,4 & 2,4 & 2,2 \\
\hline 1954 & 21374 & 13,1 & 2,6 & 5,1 \\
\hline 1955 & 19813 & 13,6 & 2,5 & 5,4 \\
\hline 1956 & 19886 & 7,5 & 2,7 & 2,8 \\
\hline 1957 & 21796 & 10,6 & 2,5 & 4,2 \\
\hline 1958 & 25873 & 7,7 & 2,5 & 3,1 \\
\hline 1959 & 27591 & 6,9 & 2,6 & 2,7 \\
\hline 1960 & 27736 & 4,3 & 2,4 & 1,8 \\
\hline 1961 & 29205 & 6,5 & 2,5 & 2,6 \\
\hline 1962 & 29069 & 6,5 & 2,4 & 2,7 \\
\hline 1963 & 28781 & 6,6 & 2,4 & 2,8 \\
\hline 1964 & 30201 & 5,3 & 2,3 & 2,3 \\
\hline 1965 & 31751 & 7,2 & 2,4 & 3,1 \\
\hline 1966 & 31710 & 5,4 & 2,4 & 2,2 \\
\hline 1967 & 32623 & 5,2 & 2,4 & 2,2 \\
\hline 1968 & 33755 & 7,7 & 2,3 & 3,3 \\
\hline 1969 & 31227 & 7,0 & 2,3 & 3,0 \\
\hline 1970 & 29288 & 6,5 & 2,3 & 2,8 \\
\hline 1971 & 30609 & 5,6 & 2,3 & 2,4 \\
\hline 1972 & 30987 & 11,6 & 2,4 & 4,9 \\
\hline 1973 & 28703 & 14,6 & 2,3 & 6,5 \\
\hline 1974 & 26570 & 9,4 & 2,3 & 4,1 \\
\hline 1975 & 27757 & 16,9 & 2,3 & 7,2 \\
\hline 1976 & 29996,5 & 13,3 & 2,4 & 5,6 \\
\hline 1977 & 31385 & 12,7 & 2,5 & 5,1 \\
\hline 1978 & 32787 & 14,0 & 2,6 & 5,3 \\
\hline 1979 & 34485 & 10,4 & 2,9 & 3,6 \\
\hline 1980 & 37306 & 10,5 & 2,9 & 3,6 \\
\hline 1981 & 34648,5 & 11,8 & 3,0 & 4,0 \\
\hline 1982 & 32459,5 & 16,6 & 3,1 & 5,3 \\
\hline 1983 & 36606,5 & 15,6 & 3,3 & 4,8 \\
\hline 1984 & 40785,5 & 14,2 & 3,3 & 4,3 \\
\hline 1985 & 42777 & 15,0 & 3,4 & 4,4 \\
\hline
\end{tabular}


Tableau A.2 (suite). Population moyenne sous écrou, taux de suicide en prison et taux de suicide en population générale masculine de 15 à 59 ans (p. 10000), France métropolitaine, 1945-2010

\begin{tabular}{|l|c|c|c|c|}
\hline \multicolumn{1}{|c|}{ Période } & $\begin{array}{c}\text { Population moyenne } \\
\text { sous écrou } \\
(1)\end{array}$ & $\begin{array}{c}\text { Taux de suicide } \\
\text { des personnes } \\
\text { sous écrou (2) }\end{array}$ & $\begin{array}{c}\text { Taux de suicide } \\
\text { en population générale } \\
\text { Hommes 15-59 ans (3) }\end{array}$ & $\begin{array}{c}\text { Sursuicidité des } \\
\text { personnes sous écrou } \\
(2) /(3)\end{array}$ \\
\hline 1986 & 45155,5 & 14,0 & 3,4 & 4,1 \\
1987 & 48511 & 12,4 & 3,3 & 3,8 \\
1988 & 47154,5 & 15,9 & 3,1 & 5,1 \\
1989 & 44447 & 13,9 & 3,1 & 4,5 \\
1990 & 45536,5 & 12,7 & 3,1 & 4,2 \\
1991 & 47636,5 & 13,6 & 3,1 & 4,4 \\
1992 & 48138,5 & 19,5 & 3,2 & 6,1 \\
1993 & 49202 & 19,9 & 3,4 & 5,8 \\
1994 & 50931,5 & 19,8 & 3,4 & 5,8 \\
1995 & 52140,5 & 20,5 & 3,3 & 6,3 \\
1996 & 52149 & 25,9 & 3,1 & 8,2 \\
1997 & 51192 & 24,4 & 3,1 & 7,9 \\
1998 & 50208 & 23,3 & 2,9 & 8,0 \\
1999 & 48860,5 & 24,8 & 2,7 & 9,0 \\
2000 & 46333,5 & 25,3 & 2,9 & 8,6 \\
2001 & 44968,5 & 21,8 & 2,8 & 7,7 \\
2002 & 48573 & 24,3 & 2,9 & 8,5 \\
2003 & 53591 & 21,8 & 2,9 & 7,4 \\
2004 & 55328,5 & 19,9 & 2,9 & 6,9 \\
2005 & 55467,5 & 21,1 & 2,8 & 7,7 \\
2006 & 55963,5 & 16,1 & 2,7 & 6,0 \\
2007 & 57881 & 16,4 & 2,6 & 6,3 \\
2008 & 60572 & 18,7 & 2,6 & 7,1 \\
2009 & 61553 & 19,7 & 2,7 & 7,1 \\
2010 & 61949 & 18,9 & 2,7 & \\
\hline Sources : (1) & et (2) ministère de la Justice, DAP $;(3)$ Ined jusqu'en 1999 puis CépiDc (calcul des auteures). \\
\hline
\end{tabular}


Annexe A.3. Évolution annuelle du taux de suicide en population générale masculine depuis 1925 en France métropolitaine selon différents indicateurs, p. 10000

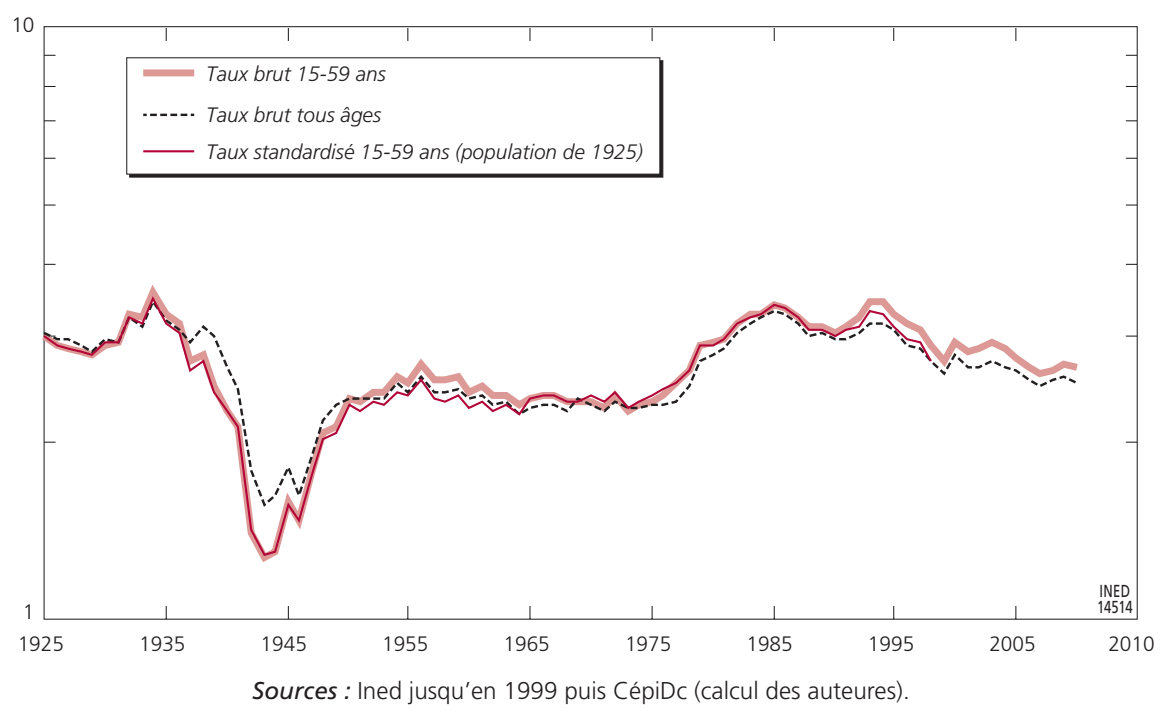




\section{RÉFÉRENCES}

Aebi M., Delgrande N., 2010, Council of Europe. Annual Penal Statistics. SPACE 1, $111 \mathrm{p}$.

AlBRAND L., 2009, La prévention du suicide en milieu carcéral, Rapport au garde des Sceaux, $310 \mathrm{p}$.

Aouba A., Péquignot F., CAmelin L., Jougla E., 2011, « Évaluation de la qualité et amélioration de la connaissance des données de mortalité par suicide en France métropolitaine, 2006 », Bulletin d'épidémiologie hebdomadaire, n 47-48, p. 497-500.

ASHRAF H., 1999, « Suicides in UK prisons increase in the past 10 years », The Lancet, 354(9176), p. 404.

Aubusson DE CAVARLAY B., 2009, « Note sur la sursuicidité carcérale en Europe : du choix des indicateurs », Champ pénal. Confrontations, DOI : 10.4000/champpenal.7558

BAJOS N., BOZON M., 2008, « Les agressions sexuelles en France : résignation, réprobation, révolte », in Bajos N., Bozon M. (dir.), Enquête sur la sexualité en France. Pratiques, genre et santé, Paris, La Découverte, p. 381-407.

Bourgoin N., 1993a, « Le suicide en milieu carcéral », Population, 48(3), p. 609-626.

BOURGOIN N., 1993b, « La mortalité par suicide en prison », Revue d'épidémiologie et de santé publique, 41(2), p. 146-154.

BOURGOIN N., 1993c, « Le suicide en prison. L'exemple du criminel contre un proche », Revue de science criminelle et de droit pénal comparé, $\mathrm{n}^{\circ} 3$, p. 575-580.

BOURGOIN N., 1994, Le suicide en prison, Paris, L'Harmattan, Logiques sociales, 271 p.

Brugha T. S., Morgan Z., Bebbington P., Jenkins R., LeWis G. et al., 2003, «Social support networks and type of neurotic symptom among adults in British households », Psychological Medicine, 33(2), p. 307-318.

CASSAN F., TOUlEMON L., KENSEY A., 2000, « L'histoire familiale des hommes détenus », Insee première, $\mathrm{n}^{\circ} 706,4 \mathrm{p}$.

Chapireau F., 2007, « La mortalité des malades mentaux hospitalisés en France pendant la deuxième guerre mondiale », Paris, Ined, Document de travail n 146, 44 p.

Chesnais J.-C., 1976, « Le suicide dans les prisons », Population, 31(1), p. 73-85.

CheSnAis J.-C., VAllin J., 1981, « Le suicide et la crise économique », Population et sociétés, $\mathrm{n}^{\circ} 147,4 \mathrm{p}$.

COLDEFy M. (dir.), 2007, La prise en charge de la santé mentale. Recueil d'études statistiques, Paris, La Documentation française, Études et statistiques, 314 p.

COLDEFy M., EXERTIER A., 2007, « La prise en charge de la santé mentale des détenus en 2003 », in Coldefy M. (dir.), La prise en charge de la santé mentale. Recueil d'études statistiques, Paris, La Documentation française, Études et statistiques, 314 p.

COldefy M., FAURe P., PRieto N., 2002, « La santé mentale et le suivi psychiatrique des détenus », Drees, Études et résultats, $\mathrm{n}^{\circ} 181,12 \mathrm{p}$.

COUR DES COMPTES, 2014, «La santé des personnes détenues : des progrès encore indispensables », Rapport public annuel 2014. Tome 1, p. 251-290. 
DAP, 2012, «Séries statistiques des personnes placées sous main de justice 1980-2012 », PMJ5, Paris, Ministère de la Justice, $57 \mathrm{p}$.

DoOley E., 1990, « Prison suicide in England and Wales, 1972-1987 », British Journal of Psychiatry, $\mathrm{n}^{\circ} 156$, p. 40-45.

Durand C., Burtka G., Federman E., Haycox J. A., Smith J. W., 1995, « A quarter century of suicide in a major urban jail: Implications for community psychiatry », American Journal of Psychiatry, 152(7), p. 1077-1080.

Durkheim É., 1897, Le suicide. Étude de sociologie, $9^{e}$ éd., 1997, Paris, PUF, Quadrige, $516 \mathrm{p}$.

Duthé G., Hazard A., Kensey A., PAn Ké Shon J.-L., 2009, « Suicides en prison : la France comparée à ses voisins européens ", Population et sociétés, n 462, 4 p.

Duthé G., HAZARd A., Kensey A., PAN KÉ ShON J.-L., 2010, « Suicides en prison en France. Évolution depuis 50 ans et facteurs de risque », Le suicide en prison : mesure, dispositifs de prévention, évaluation, Paris, DAP, Travaux et documents n 78, p. 41-64.

Duthé G., HAzArd A., Kensey A., PAn KÉ ShOn J.-L., 2011, « Laugmentation du suicide en prison en France depuis 1945 », Bulletin d'épidémiologie hebdomadaire, $\mathrm{n}^{\circ}$ 47-48, p. 504-508.

Duthé G., Hazard A., Kensey A., PAn Ké ShOn J.-L., 2013, « Suicide among male prisoners in France: A prospective population-based study », Forensic Science International, 233(1-3), p. 273-277.

FAlissard B., Loze J.-Y., GASQUet I., DuburC A., DE BeAurePaire C. et al., 2006, «Prevalence of mental disorders in French prisons for men », BMC Psychiatry, 6(33).

FAZEL S., DANESH J., 2002, « Serious mental disorder in 23000 prisoners: A systematic review of 62 surveys », The Lancet, 359(9306), p. 545-550.

FAZEL S., BAILlARGEON J., 2011, « The health of prisoners », The Lancet, 377(9782), p. 956-965.

FAzel S., CARTwright J., Norman-NotT A., HaWton K., 2008, « Suicide in prisoners: A systematic review of risk factors », Journal of Clinical Psychiatry, 69(11), p. 1721-1731.

FAZEL S., GRANn M., Kling B., HaWton K., 2010, « Prison suicide in 12 countries: An ecological study of 861 suicides during 2003-2007 », Social Psychiatry and Psychiatric Epidemiology, 46(3), p. 191-195.

Frottier P., Frühwald S., Ritter K., Eher R., SchWÄrzler J., Bauer P., 2002, « Jailhouse blues revisited », Social Psychiatry and Psychiatric Epidemiology, 37(2), p. 68-73.

Fruehwald S., Frottier P., Matschnig T., Eher R., 2003, « The relevance of suicidal behaviour in jail and prison suicides », European Psychiatry, 18(4), p. 161-165.

Fruehwald S., Frottier P., Ritter K., Eher R., Gutierrez K., 2002, « Impact of overcrowding and legislational change on the incidence of suicide in custody experiences in Austria, 1967-1996 », International Journal of Law and Psychiatry, 25(2), p. 119-128.

Fruehwald S., Matschnig T., Koenig F., Bauer P., Frottier P., 2004, « Suicide in custody. Case-control study ", British Journal of Psychiatry, 185, p. 494-498.

Guillonneau M., 2002, "Suicides en détention et infractions pénales », Cahiers de démographie pénitentiaire et criminologique, $\mathrm{n}^{\circ} 12,4 \mathrm{p}$.

HARVEY J., LiEbling A., 2001, « Suicide et tentatives de suicide en prison : vulnérabilité, ostracisme et soutien social », Criminologie, 34(2), p. 57-83.

Hawton K., VAn HeEringen K., 2009, "Suicide », The Lancet, 373(9672), p. 1372-1381. 
HAZARD A., 2008, « Baisse du suicide en prison depuis 2002 », Cahiers d'études pénitentiaires et criminologiques, $\mathrm{n}^{\circ} 22,6 \mathrm{p}$.

Hosmer D. W., Lemeshow JR. S., MAY S., 2008, Applied Survival Analysis: Regression Modeling of Time to Event Data, $2^{\text {nd }}$ Edition, Wiley, $416 \mathrm{p}$.

Humber N., Piper M., Appleby L., Shaw J., 2011, « Characteristics of and trends in subgroups of prisoner suicides in England and Wales », Psychological Medicine, 41(11), p. 2275-2285.

JoukAmAA M., 1997, «Prison suicide in Finland, 1969-1992 », Forensic Science International, 89, p. 167-174.

Kariminia A., Butler T. G., Corben S. P., Levy M. H., Grant L. et al., 2007, « Extreme cause-specific mortality in a cohort of adult prisoners - 1988 to 2002: A data-linkage study », International Journal of Epidemiology, 36(2), p. 310-316.

KENSEY A., 1991, « Suicides et tentatives de suicide en milieu carcéral de 1986 à 1990 », Bulletin du Comité de liaison des associations socioéducatives de contrôle judiciaire, $\mathrm{n}^{\circ} 24$.

KENSEY A., BENAOUdA A., 2011, « Les risques de récidive des sortants de prison. Une nouvelle évaluation ", Cahiers d'études pénitentiaires et criminologiques, $\mathrm{n}^{\circ} 36$, $8 \mathrm{p}$.

Kensey A., CASSAN F., TOUlEMON L., 2000, « La prison : un risque plus fort pour les classes populaires ", Cahiers de démographie pénitentiaire, n 9, 4 p.

LAAnAni M., Jougla E., GHOSN W., Rey G., 2013, « Variation de la mortalité par suicide selon le taux de chômage de 2000 à 2010, France métropolitaine ", Congrès ADELF-SFSP, 18 octobre 2013, Bordeaux.

LiEBLING A., 1993, « Suicides in young prisoners: A summary », Death Studies, 17(5), p. 381-407.

LIEBLING A., 1995, «Vulnerability and prison suicide », British Journal of Criminology, 35(2), p. 173-187.

Mamelet M.-R., 1978, « La politique de secteur », Projet, n 125, p. 588-598.

MarCus P., AlCABES P., 1993, "Characteristics of suicides by inmates in an urban jail », Hospital and Community Psychiatry, 44(3), p. 256-261.

Marzano L., Hawton K., Rivlin A., FAzel S., 2011, « Psychosocial influences on prisoner suicide: A case-control study of near-lethal self-harm in women prisoners ", Social Science \& Medicine, 72(6), p. 874-883.

MouqueT M.-C., 2005, « La santé des personnes entrées en prison en 2003 », Drees, Études et résultats, $\mathrm{n}^{\circ} 386,12 \mathrm{p}$.

Mucchielli L., 2002, " Les homicides », in Mucchielli L., Robert P. (dir.), Crime et sécurité. L'état des savoirs, Paris, La Découverte, p. 148-157.

O'DRISCOll C., SAMUEls A., ZaCKA M., 2007, « Suicide in New South Wales prisons, 1995-2005: Towards a better understanding ", Australian \& New Zealand Journal of Psychiatry, 4l(6), p. 519-524.

OMS, 2007, Preventing Suicide in Jails and Prisons, Genève, Organisation mondiale de la santé, $28 \mathrm{p}$.

OrRo D., SENGHOR H., 2010, «Confrontations d'analyses multivariées portant sur un événement rare : le suicide carcéral. Rapport interne ", Paris, Ined, 61 p.

PAN KÉ SHON J.-L., 2013, « Suicides en situation d'enfermement au début du XXI ${ }^{e}$ siècle. Approche compréhensive à partir de la dernière lettre des suicidés en prison », Sociologie, 2(4), $37 \mathrm{p}$.

Shaw J., Baker D., Hunt I., Moloney A., Appleby L., 2004, « Suicide by prisoners. National clinical survey », British Journal of Psychiatry, 184, p. 263-267. 
TERRA J.-L., 2003, Prévention du suicide des personnes détenues, Rapport de mission au ministre de la Justice et au ministre de la Santé, de la famille et des personnes handicapées, $218 \mathrm{p}$.

TOURnier P., Chemithe P., 1979, « Contribution statistique à l'étude des conduites suicidaires en milieu carcéral 1975-1978 », Centre national d'études et recherches pénitentiaires (CNERP), Ministère de la Justice, DAP, 81 p.

TOWL G., CRIGHTON D., 1998, « Suicide in prisons in England and Wales from 1988 to $1995 »$, Criminal Behaviour and Mental Health, n 8, p. 184-192.

VALlin J., MeSLÉ F.,1988, Les causes de décès en France de 1925 à 1978, Paris, Ined/ PUF, Cahier n 115, 607 p.

VON BUeltZingSLOEWEN I., 2007, L'Hécatombe des fous. La famine dans les hôpitaux psychiatriques sous l'Occupation, Paris, Aubier/Flammarion, 520 p.

WAy B., Miraglia R., SAWyer D., BEER R., EDDy J., 2005, « Factors related to suicide in New York state prisons ", International Journal of Law and Psychiatry, 28(3), p. 207-221. 


\section{Géraldine Duthé, Angélique HAZARD, Annie KenSEY • SUICIDE DES PERSONNES ÉCROUÉES EN FRANCE : ÉVOLUTION ET FACTEURS DE RISQUE}

Le niveau de suicide en prison est actuellement élevé en France : 18,5 suicides pour 10000 personnes écrouées pendant la période 2005-2010, soit sept fois plus qu'en population générale. Cet article présente l'évolution du suicide depuis le milieu du XIX ${ }^{\mathrm{e}}$ siècle, mettant en avant une spécificité croissante du suicide en milieu carcéral par rapport à la population générale. L'analyse porte ensuite sur les associations qui existent entre les risques de suicide et les caractéristiques individuelles, à partir de la base de gestion de l'administration pénitentiaire qui regroupe l'ensemble des séjours sous écrou observés en France entre le $1^{\text {er }}$ janvier 2006 et le 15 juillet 2009. Sur cette période, près de 378000 séjours ont été observés (correspondant à plus de 221000 personnes-années) et 378 suicides ont été identifiés. Les résultats confirment la vulnérabilité des personnes en détention provisoire et montrent le rôle prépondérant de l'isolement, qu'il soit physique ou social, ainsi que celui de la nature de l'infraction qui a motivé la mise sous écrou. Si l'amélioration de l'environnement du détenu est un point mis en avant dans les nouvelles politiques de prévention, la nature de l'infraction, comme facteur de risque suicidaire, devrait également être prise en compte.

\section{Géraldine Duthé, Angélique HAZARD, Annie KENSEY • TRENDS AND RISK FACtors fOR} PRISONER SUICIDE IN FRANCE

France currently has a very high level of prisoner suicides. Between 2005 and 2010, the mean annual rate was 18.5 suicides per 10,000 prisoners seven times higher than for the general population. In this article, we begin by describing how suicide has changed since the mid-nineteenth century, highlighting the increasingly specific nature of suicide among prisoners versus non-prisoners. We then analyse the associations between individual characteristics and suicide risk, based on information from the French prison service's database on all prison terms served in France between 1 January 2006 and 15 July 2009. Over this period, nearly 378,000 terms were observed (corresponding to more than 221,000 person-years) and 378 suicides were identified. Our results confirm the vulnerability of pre-trial detainees, and show that isolation, be it physical or social, is a key component of suicide risk, along with the type of offence resulting in a prison sentence. Improving the conditions of detention is one of the priorities of prevention policies now being implemented, but the nature of the offence must also be taken into account as a suicide risk factor.

\section{Géraldine DUthé, Angélique HAZARD, Annie KENSEY • SUICIDIO DE PERSONAS ENCARCELADAS EN FRANCIA: EVOLUCIÓN Y FACTORES DE RIESGO}

Actualmente, en Francia, la frecuencia del suicidio en prisión es elevada: 18,5 suicidios por 10000 personas encarceladas durante el periodo 2005-2010, es decir, siete veces más que en la población general. Este artículo presenta la evolución del suicidio desde mediados del siglo XIX, y subraya una especificidad creciente del suicidio en prisión respecto al observado en la población general. Se estudian a continuación asociaciones entre el riesgo de suicidio y características individuales, a partir de los datos de la base de gestión de la administración penitenciaria que reúne todas las estancias en prisión observadas en Francia entre el $1^{\circ}$ de enero de 2006 y el 15 de Julio de 2009. Durante este periodo, fueron observadas cerca de 378000 estancias (correspondiendo a más de 221000 personas-año) durante las cuales se produjeron 378 suicidios. Los resultados confirman la vulnerabilidad de las personas en detención provisional y muestran el papel preponderante del aislamiento, físico o social, así como el de la naturaleza de la infracción que ha provocado el encarcelamiento. Si las nuevas políticas de prevención se proponen mejorar el entorno del detenido, también debería tomarse en cuenta la naturaleza de la infracción come factor de riesgo.

Mots-clés : Mortalité, suicide, prison, France, sursuicidité, risques, modèle de durée.

Keywords: Mortality, suicide, prison, France, excess suicide mortality, hazard, duration model. 